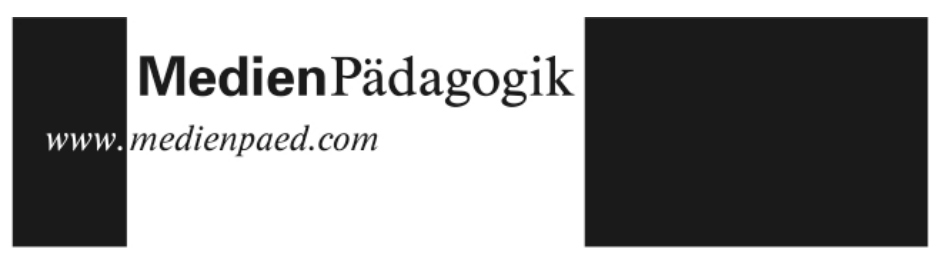

Rezensionen

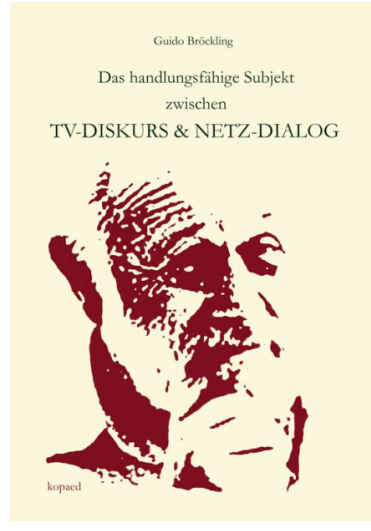

Guido Bröckling

Das handlungsfähige Subjekt zwischen TV-Diskurs \& Netz-Dialog

München: kopaed, 2012. 279 Seiten

ISBN: $978-3-867-36-280-1$

$€ 18.80$; CHF 22.70

\title{
Zwischen Kommunikologie und Medienkompetenz. Vilém Flusser weitergedacht.
}

Ziel der von Guido Bröckling als Dissertation vorgelegten Arbeit ist es, in Bezug auf die theoretischen Ideen Vilém Flussers ein handlungsorientiertes Medienbildungskonzept zu erarbeiten. Das bedeutet, dass er von den Perspektiven, die Flusser für seine Arbeit gesetzt hat - einmal das Medium im Kontext seiner Sozialität und zum anderen das Subjekt in Relation zum Medium zu betrachten - vor allem die zweite Sichtweise betont. Nun ist allerdings Medienbildung bezogen auf den theoretischen Ansatz von Flusser nicht allein auf den Umgang mit Medien zu beschränken. Die Idee, die Flusser mit seinem Modell des Netzdialogs entwickelt, geht einen Schritt weiter. Er weist den Medien in der Gestaltung des Netzdialoges eine den Menschen gleichberechtigte Funktion zu. Bröckling nimmt diese Blickrichtung ein und rekonstruiert Flussers Ansätze unter Einbezug weiterer Autoren, auf die sich auch Flusser selbst bezieht. Von größerer Bedeutung hierbei ist Martin Buber, der sich mit dem Verhältnis von Ich und Wir auseinandergesetzt hat, also der Konstitution des Menschseins innerhalb der Gesellschaft und wiederum der Konstitution der Gesellschaft unter dem Aspekt des sich des Wir bewussten Individuums. Ebenfalls von Bedeutung sind für inn die Kommunikationstheorien bzw. theoretischen Ansätze von Jürgen Habermas, der sich ja ebenso wie Flusser mit der Kommunikation und ihren Gesetzmäßigkeiten, aber auch und vor allen deren Strukturen auseinandergesetzt und dies in Beziehung zur Entwicklung der Gesellschaft gebracht hat. 
Im Zentrum der Arbeit steht die Aufarbeitung des Gedankengebäudes Vilém Flussers. Bröckling nimmt zentrale Begriffe auf und bestimmt diese unter dem Leitmotiv seiner Fragestellung. Der erste Bereich den er herausarbeitet ist der medienkulturelle Wandel und die darauf basierenden Wirklichkeitskonstruktionen. Der Medienwandel mündet in seiner Beschreibung in einer Neubewertung der Medien auf Grund eines selbst bestimmten Umganges mit diesen. Dieser Grundgedanke, dass Medienbildung zu einem selbstbestimmten und auch konstruktiv-reflexiven Umgang mit den Medien führen soll und muss, ist wohl auch der Extrakt der Flusserschen Philosophie und der beiden zentralen Dimensionen einer Philosophie, die Bröckling in Anlehnung an Flusser als TV-Diskurs und Netz-Dialog bezeichnet. In zwei Szenarios, die Bröckling darauf aufbauend entwickelt, setzt er den TV-Diskurs als bestimmendes Moment einer idealtypischen Massenmedienrealität in Relation und in den Gegensatz zum Netz-Dialog. Der TV-Diskurs sei kommunikativ einheitlich und bestimme zwingend den gesellschaftlichen Dialog durch Eliten, die in diesem Diskurs der Mehrheit ihre Weltanschauung oktroyieren. Das anzustrebende Ideal, das durch die Digitalisierung erreicht werden könne, laute, den Menschen aus der Programmierung dieses der Masse dienenden TV-Diskurses zu befreien und zur Auseinandersetzung mit anderen im Netz-Dialog zu befähigen.

Im Szenario des Netz-Dialoges rekonstruiert Bröckling das Modell von Flusser, einer Dialogstruktur im Weiteren als kommunikative Struktur. Dabei bezieht er sich auf die jeweils genannten Pole des Ich und Wir, und hierbei auf Buber bzw. das interaktionistische Modelle von Mead mit dem I und Me. Im Idealfall des Szenarios des Netz-Dialoges, den er als einen telematischen beschreibt, werde jenseits der personalen Interaktion eine telematische erzeugt. «Identitätskonstruktion bedeutet Informationsprozess» (116). Dieser grundlegende Gedanke verfolgt er weiter, indem er die Möglichkeiten einer gesellschaftlichen Utopie, in der das Ich und das Wir aufgehoben sind, in der Zuwendung zum telematischen Prozess gesehen werden, in der Einbindung der Telematik und des TV-Diskurses in die Konstruktion einer Utopie. Notwendigerweise hat in seinem Modell und er weist darauf hin, dass er den Ansatz von Buber in eine angewandte Telematik überführt, die Netzstruktur mit ihren Implikaten eine zentrale Funktion. Der Vielfalt und vielfälti- 
ge Möglichkeiten von Information und Kommunikation wird hier ein von ihm auch so genannter Zensor zugefügt. Ohne ausgrenzende bzw. diffamierende Bewertungen vorzunehmen, sorge dieser dafür, dass die von den Subjekten gespeisten Programme und Informationen so gestaltet und geordnet sind, dass sie zu einer Erhöhung der Informationen und der Informationsmöglichkeiten beitragen könnten. Die Voraussetzung dafür allerdings sei, dass die Informationseingabe und die kommunikative Auseinandersetzung innerhalb des Netzdialoges nicht mehr wie im TV-Diskurs zu einer einseitig beeinflussten Veränderung führen soll, sondern eine quasi spielerische ist. Hier wird dem Homo Faber der Homo Ludens entgegengesetzt. Das Ideal einer telematischen Gesellschaft ist eine, in der unter dem Aspekt des Spielerischen die Gesellschaft konstruiert und vor allen konstituiert wird. Hier finden wir Anklänge an viele Utopien, vom frühen Marx bis hin zu literarischen Auseinandersetzungen, etwa dem Glasperlenspiel von Hermann Hesse.

Der letzte Teil der Arbeit stellt in gewisser Weise einen Bruch dar, weil der Autor sich nun der Ausformulierung seiner Vorstellung von Medienbildung widmet. Dieser Bruch ist notwendig, da an dieser Stelle in den Worten des Autors «weitergedacht» und Kommunikologie und Medienbildung zusammengebracht werden. Hier verweist Bröckling auch und unter Bezug auf Konzepte der Medienkompetenz darauf, welche Fähigkeiten entwickelt werden und unterstützt werden sollen, um eine kommunikologische oder besser sozio- und medienkulturelle Kompetenz zu entwickeln - als Grundlage des Ludere im Netz. Die telematische Gesellschaft beruhe eben nicht mehr auf Arbeit, die sie abschafft, sondern auf einer Spielkompetenz. In der Konkretion ist diese Spielkompetenz allerdings sehr nah am Konzept der Medienkompetenz, wie wir sie aus der einschlägigen Literatur kennen. In diesem Sinne konstituiert Medienbildung sich unter kommunikologischen Aspekten als ausformulierte Medienkompetenz und der Autor kann sich wieder auf Theoretiker beziehen, die er in sein Gesamtmodell eingebracht hat. Inwieweit allerdings die Ausbildung von Medienkompetenz in der Lage ist, das Ideal einer telematischen Utopie mit zu schaffen, das bleibt in der Arbeit offen. Ebenso offen bleibt die Frage wie sich denn eine Idealgesellschaft, die sich der digitalen Möglichkeiten des Denkens und denkenden Handelns bedient, ohne Arbeit tragen soll und inwieweit eine solche Utopie davon 
abstrahieren kann, dass sich die Techniken - wie Bröckling in Anlehnung an Flusser sehr wohl in der Auseinandersetzung mit dem TV-Diskurs feststellt aus elitären Interessen entwickelt haben und dass hinter innen weiterhin Prinzipien von Macht und Herrschaft dominieren. Inwieweit solche Prinzipien aufzulösen sind, in einem Modell des Homo Ludens, inwieweit solche Prinzipien nicht sogar einem Modell des Homo Faber entgegenstehen, dieser Frage wäre weiter nachzugehen.

Die an die Arbeit zu stellenden kritischen Fragen beeinträchtigen nicht ihren Wert. Er liegt vor allen Dingen darin, dass es dem Autor überzeugend gelingt, die theoretischen Ansätze von Flusser - die sehr häufig aus Gedankensplittern bestehen und in der Regel nicht ausformuliert sind, ja sich sogar widersprechen - unter Bezug auf andere Philosophen und Denker zusammenzuführen, so diese sich in den Prospekt einer zukünftigen Mediengesellschaft und vor allen einer Bildung zur Gestaltung dieser Gesellschaft einbringen lässt. Gut wäre es gewesen, wenn der Autor seine Konstruktion, die hin zur Medienbildung führt, in der Weise nachvollziehbar gemacht hätte, dass er seine Gedankenbündel am Ende der einzelnen Kapiteln zusammenfasst und auf dieser Basis die jeweilige Weiterführung eingeleitet hätte. So ist der Versuch den roten Faden der Arbeit nachzuvollziehen, sehr schwierig. Unklar ist, warum so viele Bereiche in großer Ausführlichkeit dargestellt wurden, aber nur recht knapp in die Gestaltung eines Konzeptes von Medienbildung eingehen. Hier hätte der Autor dem Leser eine Hilfe geben sollen, allein schon um die wertvollen Gedanken dieser Arbeit nachzuvollziehen.

Bernd Schorb 